\title{
The Weak Stable Sets for Fuzzy Cooperative Games
}

\author{
Shuli Wang \\ College of Science, Yanshan University, Qinhuangdao 066004, China \\ E-mail:sunglow_wind@hotmail.com \\ Ning Jiang \\ College of Science, Yanshan University, Qinhuangdao 066004, China
}

\begin{abstract}
In this paper, by expanding the definition of dominance, we present the new concepts of weak core and weak stable set for fuzzy cooperative game, and study the properties of them.
\end{abstract}

Keywords: Fuzzy game, Weak dominance, Core, Weak stable set

\section{Introduction}

Since J.V. Neumann and O. Morgenstern introduced cooperative game in 1944(Neumann and Morgenstern, 1944), cooperative game has received a generous concern from scholars and become a research focus in academia. Half a century has witnessed the formation of the mature theory of cooperative game. As an emerging branch of game theory, fuzzy game theory started with work of Aubin in 1974(Aubin, 1974, pp. 891-984), where special attention is paid to the core concept. The purpose of this paper is to give the new notion of weak dominance and weak stable set for fuzzy cooperative game, and discuss the properties of them.

\section{Basic definitions}

Give a finite set $N=\{1,2, \cdots, n\}$ of players, a fuzzy coalition is a vector $d$ in $[0,1]^{n}$. The i-th coordinate $d_{i}$ of $d$ is called the participation level of player $i$ in the fuzzy coalition $d$. Instead of $[0,1]^{n}$, we also write $F^{N}$ for the set of fuzzy coalitions. The fuzzy coalition $e^{N}=(1,1, \cdots, 1)$ is called the grand coalition, and the fuzzy coalition (the $n$-dimensional vector) $O=(0,0, \cdots, 0)$ corresponds to the empty crisp coalition. From now on we use the notation $K(d)=\left\{i \in N: d_{i}>0\right\}$.

A fuzzy cooperative game with player set $N$ is a function $v: F^{N} \rightarrow R$ with the property $v(O)=0$, assigning to each fuzzy coalition the value achieved by cooperation. The set of fuzzy games with player set $N$ will be denoted by $F G^{N}$.

Let $v \in F G^{N}$ and let $I(v)$ be the imputation set of $v$, i.e.

$$
I(v)=\left\{x \in R^{n}: \sum_{i \in N} x_{i}=v\left(e^{N}\right), x_{i} d_{i} \geq v(d \mid i), \forall d \in[0,1]^{n}\right\}
$$

Here $d \mid i=\left(0,0, \cdots, d_{i,}, \cdots 0\right)$.

The core of a fuzzy game $v$ is the subset of imputations which are stable against any possible deviation by fuzzy coalitions (Tijs, Branzei \& Ishihara, 285-296), i.e.

$$
C(v)=\left\{x \in R^{N} \mid \sum_{i \in N} x_{i}=v\left(e^{N}\right), \sum_{i \in N} d_{i} x_{i} \geq v(d), d \in F^{N}\right\} .
$$

Now we give the new definitions.

Definition 1. Let $v$ be a fuzzy game, $x, y \in I(v)$ and $d \in F^{N}(d \neq 0)$, we say $x$ weakly dominates $y$ through $d$, denoted $x \succ_{d}^{w} y$, if

(1) $\sum_{i \in K(d)} d_{i} x_{i}>\sum_{i \in K(d)} d_{i} y_{i}$;

(2) $\sum_{i \in N} d_{i} x_{i} \leq v(d)$. 
We simply say $x$ weakly dominates $y\left(x \succ^{w} y\right)$ if there exists a fuzzy coalition sequence $d$ such that $x \succ_{d}^{w} y$.The negation of $x \succ^{w} y$ is denoted here by $x \succ^{w} y$.

The difference of weak dominance and dominance is $\sum_{i \in K(d)} d_{i} x_{i}>\sum_{i \in K(d)} d_{i} y_{i}$ which weakens the condition $x_{i}>y_{i}$ foe every $i \in K(d)$. Obviously, if $x \succ y$ then $x \succ^{w} y$, but the contrary is uncertain.

The weak dominance core of the fuzzy game $v$ is the set of imputations which are not weakly dominated by any other imputation, i.e.

$$
Đ C(v)=\left\{x \in I(v) \mid y \succ^{w} x, \forall y \in I(v)\right\} .
$$

Definition 2. The weak stable set of a fuzzy game $v$ is a nonempty set $T$ of imputations satisfying the properties:

(1)(Internal Stability) $x \succ^{w} y$ for every $x, y \in T$;

(2)(External Stability) For every $z \in I(v) \backslash T$, there is an imputation $x \in T$ such that $x \succ^{w} z$.

\section{The weak sable sets for fuzzy games}

Lemma Let $v$ be a fuzzy game and $x, y \in I(v)$. We have $x \succ^{w} y$ if and only if there exists a coalition $d \in F^{N}$ and

$\sum_{i \in N} d_{i} y_{i}<v(d)$.

Proof: It is obvious that if $x \succ^{w} y$ then we have $\sum_{i \in N} d_{i} y_{i}<v(d)$.

On the contrary hand, if $d \in F^{N}$ and $\sum_{i \in N} d_{i} y_{i}<v(d)$, then there exists $\varepsilon$ such that $\sum_{i \in N} d_{i} y_{i} \leq v(d)-\varepsilon$, that is $\sum_{i \in N} d_{i} y_{i}+\varepsilon \leq v(d)$.Therefore, we have a coalition $x$ such that $\sum_{i \in K(d)} d_{i} x_{i}>\sum_{i \in K(d)} d_{i} y_{i}$, where $x_{i} \geq y_{i}$. Hence, $x \succ_{d}^{w} y$.

Theorem 1. If $v$ is a fuzzy game, then $D C(v) \subset D C(v)$.

Proof: Let $\forall y \in D C(v)$.If $y \notin D C(v)$, then there exist $d \in F^{N}$ and $x \in I(v)$ such that $\forall i \in K(d), x_{i}>y_{i}$ and $\sum_{i \in N} d_{i} x_{i} \leq v(d)$.So we have $\sum_{i \in K(d)} d_{i} x_{i}>\sum_{i \in K(d)} d_{i} y_{i}$, and $x \succ_{d}^{w} y$. This is a contradiction. Hence $y \in D C(v)$.

Theorem 2. Let $x \in I(v) \cdot x \in D C(v)$ if and only if for all $d \in F^{N}$ we have

$\sum_{i \in N} d_{i} x_{i} \geq v(d)$

Proof: If for all $d \in F^{N}$ we have $\sum_{i \in N} d_{i} x_{i} \geq v(d)$, then $x \in D C(v)$. Otherwise there exist a coalition $d \in F^{N}$ and $y \in I(v)$ such that $y \succ_{d}^{w} x$. Hence we have $\sum_{i \in K(d)} d_{i} y_{i}>\sum_{i \in K(d)} d_{i} x_{i}$ and $\sum_{i \in N} d_{i} y_{i} \leq v(d)$, then $\sum_{i \in N} d_{i} x_{i} \leq v(d)$. This is contradict with (2).

If $x \in D C(v)$, we have the fact all $d \in F^{N}$ satisfy (2). Otherwise, if $\sum_{i \in N} d_{i} x_{i}<v(d)$ for a coalition $d \in F^{N}$, then there is an imputation $y \in I(v)$ such that $y \succ_{d}^{w} x$. This also is a contradiction.

From Theorem 2 we obtain the following corollary. 
Corollary The weak dominance core of a fuzzy game $v$ is the core of it, i.e.

$$
D C(v)=C(v) .
$$

Theorem 3. If $v$ is a fuzzy game, then for every weak stable set $T, C(v)=\boxplus C(v) \subset F$.

Proof: Let is $F$ the sable set. Since $D C(v)$ consists of undominated imputations and each imputation in $I(v) \backslash T$ is dominated by some imputation by the external stability property, it follows that $\boxplus C(v) \subset T$.

Theorem 4. If the core $C(v)=D C(v)$ is the weak stable set of a fuzzy game $v$, then it is unique.

Proof: Suppose the core $C(v)=\emptyset C(v)$ is the weak stable set of $v$, and $K$ is the other one. By Theorem 3 we can know $C(v)=D C(v) \subset K$.It follows that $C(v)=D C(v)=K$. Because $y \in K \backslash C(v)$ implies there exist $x \in K$ and $d \in F^{N}$ such that $x \succ_{d}^{w} y$. This is contradict with the internal property of $K$.

\section{References}

Aubin, J.P. (1974). Ceur et valeur des jeux flous a paiements lateraux. Comptes. Comptes Rendus de l'Academie des Sciences, 279(A), 891-894.

Neumann, V. \& Morgenstern, O. (1944). Theory of Game and Economic Behavior. Princeton University Press.

Tijs, S., Branzei, R. \& Ishihara, S., et al. (2004). On cores and stable sets for fuzzy game. Fuzzy sets and system, 146, 285-296. 